\title{
The POINT-AGAPE survey: 4 high signal-to-noise microlensing candidates detected towards M 31
}

\author{
S. Paulin-Henriksson ${ }^{1}$, P. Baillon ${ }^{2}$, A. Bouquet ${ }^{1}$, B. J. Carr $^{3}$, M. Crézé ${ }^{1,4}$, N. W. Evans ${ }^{5,6}$, Y. Giraud-Héraud ${ }^{1}$, \\ A. Gould ${ }^{1,7}$, P. Hewett ${ }^{6}$, J. Kaplan ${ }^{1}$, E. Kerins ${ }^{8}$, Y. Le Du ${ }^{1,5}$, A.-L. Melchior ${ }^{9}$, \\ S. J. Smartt ${ }^{6}$, and D. Valls-Gabaud ${ }^{10}$ \\ (The POINT-AGAPE Collaboration) \\ ${ }^{1}$ Laboratoire de Physique Corpusculaire et Cosmologie, Collège de France, 11 place Marcelin Berthelot, \\ 75231 Paris, France \\ 2 CERN, 1211 Genève, Switzerland \\ 3 Astronomy Unit, School of Mathematical Sciences, Queen Mary, University of London, Mile End Road, \\ London E1 4NS, UK \\ ${ }^{4}$ Université Bretagne-Sud, campus de Tohannic, BP 573, 56017 Vannes Cedex, France \\ 5 Theoretical Physics, 1 Keble Road, Oxford OX1 3NP, UK \\ ${ }^{6}$ Institute of Astronomy, Madingley Road, Cambridge CB3 OHA, UK \\ 7 Department of Astronomy, Ohio State Univ., 140 West 18th Avenue, Columbus, OH 43210, USA \\ 8 Astrophysics Research Institute, Liverpool John Moores Univ., 12 Quays House, Egerton Wharf, \\ Birkenhead CH41 1LD, UK \\ 9 LERMA, FRE2460, Obs. de Paris, 61 avenue de l'Observatoire, 75014 Paris, France \\ 10 Laboratoire d'Astrophysique UMR CNRS 5572, Obs. Midi-Pyrénées, 14 avenue Édouard Belin, \\ 31400 Toulouse, France
}

Received 1 July 2002 / Accepted 3 April 2003

\begin{abstract}
We have carried out a survey of the Andromeda galaxy for unresolved microlensing (pixel lensing). We present a subset of four short timescale, high signal-to-noise microlensing candidates found by imposing severe selection criteria: the source flux variation exceeds the flux of an $R=21$ magnitude star and the full width at half maximum timescale is less than 25 days. Remarkably, in three out of four cases, we have been able to measure or strongly constrain the Einstein crossing time of the event. One event, which lies projected on the M 31 bulge, is almost certainly due to a stellar lens in the bulge of M 31 . The other three candidates can be explained either by stars in M 31 and M 32 or by MACHOs.
\end{abstract}

Key words. galaxies: halo - galaxies: individual: M 31 - gravitational lensing - dark matter

\section{Introduction}

The galactic dark matter may be partly composed of compact objects (e.g., black holes, faint stars, brown dwarfs, jupiters) that reside in halos and are popularly called MACHOs (“MAssive Compact Halo Objects"). Microlensing surveys towards M 31 (Crotts 1992; Baillon et al. 1993) have the potential to resolve the puzzling question raised by searches toward the Magellanic Clouds: the optical depth $\tau \sim 10^{-7}$ measured by MACHO (Alcock et al. 2000) is too large by a factor 5 to be accounted for by known populations of stars and too small by the same factor to account for the dark matter, while the mass scale inferred for the lenses $M \sim 0.4 M_{\odot}$ is in the mid-range of normal stars. EROS (Lasserre et al. 2000) obtained upper limits that are consistent with the MACHO results.

Send offprint requests to: S. Paulin-Henriksson, e-mail: paulin@cdf.in2p3.fr
Since M 31 is 15 times more distant than the Magellanic Clouds, the stars are about 200 times fainter and more densely packed on the sky. Even with new techniques that are required to monitor flux changes of unresolved stars in the face of seeing variations (Crotts \& Tomaney 1996; Ansari et al. 1997; Ansari et al. 1999), the low signal-to-noise ratio $(S / N)$ engenders a whole range of problems. First, the detection efficiency is reduced. Second, there is a degeneracy between the Einstein crossing time, the impact parameter and the source flux (Gould 1996). Third, some variable stars cannot be easily distinguished from microlensing events and so will contaminate the signal. We elaborate on each of these points as follows:

i) The loss of detection efficiency is severe because a microlensing event can be rejected by the selection procedure if the source star or neighbouring blended stars are variable. Indeed, if it is to be detected as microlensing, an event must rise above the photon noise due to all the blended 
neighbouring stars. For a fixed impact parameter, the brighter the source star, the easier it is to detect the event. So, bright sources are the most likely microlensing candidates. Unfortunately, the Hipparcos catalogue shows that most of the bright sources with $M_{V}<0$ are prone to intrinsic variability (Perryman et al. 1997).

ii) The degeneracy between lightcurve parameters occurs mainly around the time of maximum magnification and becomes more severe as the impact parameter becomes smaller. It can be partly broken for events with good $S / N$ and good sampling on the wings - as for three of the four events presented later.

iii) To distinguish between any MACHO population and variable stars, we intend to exploit the fact that M 31 is highly inclined $\left(i \sim 77^{\circ}\right)$ to our line of sight. Therefore, if MACHOS are distributed in a roughly spherical halo, the density of MACHOs along the line of sight is larger on the far side of the M 31 disk than on the near side. This implies a larger optical depth and an excess of microlensing events on the far side (Crotts 1992; Kerins et al. 2001).

The POINT-AGAPE collaboration is carrying out a pixellensing survey of M 31 using the Wide Field Camera (WFC) on the $2.5 \mathrm{~m}$ Isaac Newton Telescope (INT). We monitor two fields, each of $\sim 0.3 \mathrm{deg}^{2}$, located North and South of the M 31 centre. After a brief description of the observations and data analysis in Sect. 2, we present four events with high $S / N$ and short durations in Sect. 3, for which microlensing is by far the most plausible interpretation.

\section{Observations and data analysis}

The analysed data are from 143 nights between August 1999 and January 2001. The observations are made in three bands close to Sloan $g^{\prime}, r^{\prime}, i^{\prime}$. The exposure times are typically between 5 and $10 \mathrm{~min}$ per night, field and filter. Because the total allocated time per night is usually less than one hour, observations are not perfomed in all filters each night. Moreover, the observations are strongly clustered in time because the WFC was not always mounted on the telescope.

The data reduction is described in detail by Paulin-Henriksson (2002) and is similar to the method given in previous papers (Ansari et al. 1997; Aurière et al. 2001; Calchi Novati et al. 2002). After bias subtraction and flat-fielding, each image is geometrically and photometrically aligned relative to reference images (one per CCD), which are chosen to have long exposure times, typical seeing between 1'. 3 and 1.' 6 , and little contamination from the Moon. To remove the correlations with seeing variations, we first compute lightcurves on 7-pixels square "superpixels". We then apply an empirical correction on the flux of the superpixels, called "seeing stabilisation". This is described briefly in Sect. 2.1 and will be discussed in more detail in a forthcoming paper. The conversion to Johnson/Cousins $(V, R, I)$ is made by using the photometry standards of Haiman et al. (1994). The detection of events is made in the $r^{\prime}$ band, which offers the best compromise between sampling and sky background. Other bands are then used to test the achromaticity of candidates. A bump is defined by at least three consecutive $r^{\prime}$ data points

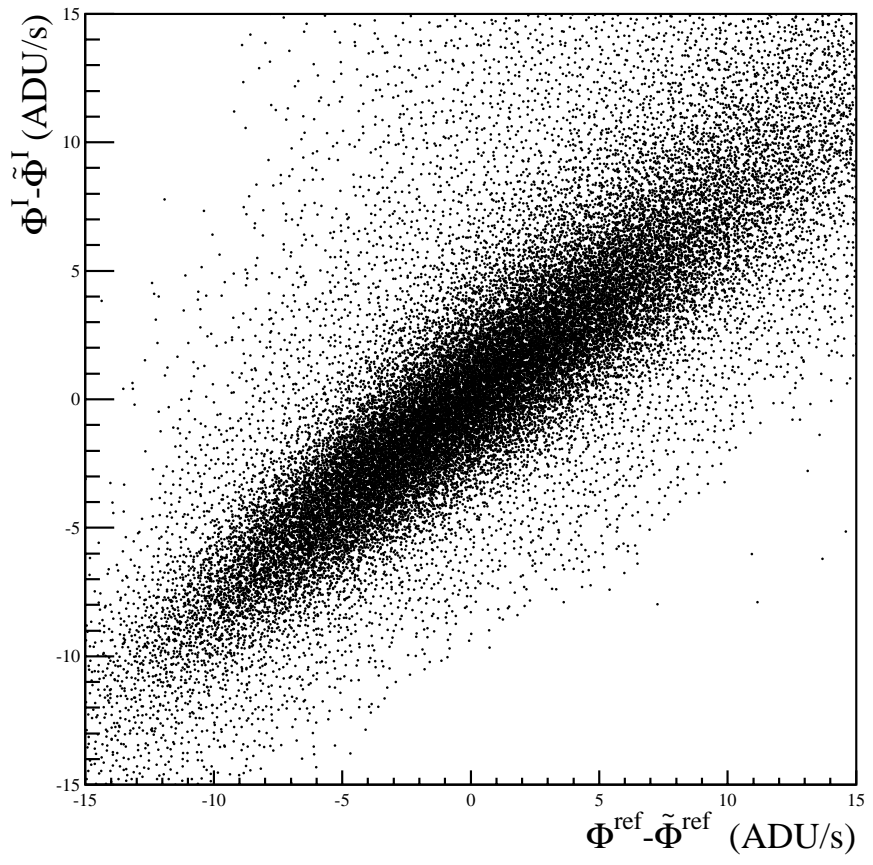

Fig. 1. Correlation between $\Phi_{i}^{I}-\widetilde{\Phi}_{i}^{I}$ and $\Phi_{i}^{\text {ref }}-\widetilde{\Phi}_{i}^{\text {ref }}$ for a seeing difference between image $I$ and the reference image: $\Delta$ seeing $\sim 0.4^{\prime \prime}$ and $(7 \times 7)$ superpixels.

rising above the baseline by at least $3 \sigma$. In this way, we detect about 80000 variable objects. As a preliminary selection, we keep a subsample of the brightest $10 \%$. More precisely, we demand $R(\Delta F)<21$, where $R(\Delta F)$ is the (Cousins) magnitude of the flux difference between the baseline flux and the maximally magnified flux during the event. Note that for small impact parameters, such as applies for the four candidates presented below, $R(\Delta F)$ is similar to the magnitude of the event at maximum magnification. Selection of the microlensing candidates among the remaining events is described in Sect. 2.2.

\subsection{Seeing stabilisation}

For very crowded fields like ours, and in the absence of resolved stars, the difference between an image and its own median comes from star density fluctuations. This difference is fully correlated from image to image. The correlation, shown in Fig. 1, is

$\Phi_{i}^{I}-\widetilde{\Phi}_{i}^{I} \approx\left(\alpha^{I}+1\right) \times\left(\Phi_{i}^{\mathrm{ref}}-\widetilde{\Phi}_{i}^{\mathrm{ref}}\right)$

where $\Phi_{i}^{I}$ and $\Phi_{i}^{\text {ref }}$ are the fluxes of superpixel $i$ on image $I$ and the reference image respectively, $\widetilde{\Phi}_{i}$ is the median flux computed on a 41-pixels (13"'53) square centred on the superpixel $i$; and $\alpha^{I}$ is constant over image $I$ (zero when the telescope conditions are identical for image $I$ and the reference image). The slope of the correlation depends on the seeing difference between the two images: the larger the seeing on image $I$, the more this image is close to its median, and the smaller the correlation parameter $\alpha^{I}$. Figure 2 shows the correlation between $\alpha^{I}$ and the seeing difference $\Delta$ seeing between the image and the 


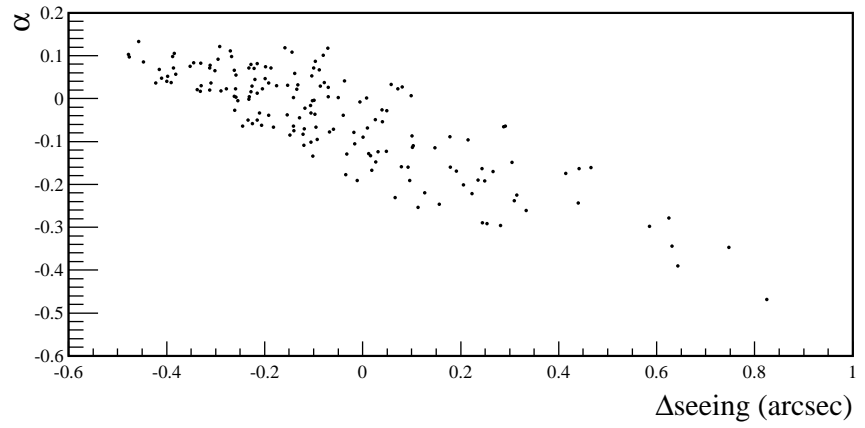

Fig. 2. The coefficient $\alpha^{I}$ versus the seeing difference between images and reference image for a sample of 166 images and $(7 \times 7)$ superpixels.

reference image. As expected, it indicates that this correlation is very strong. Therefore, the corrected flux

$\Phi_{i}^{\mathrm{C}, \mathrm{I}}=\frac{\Phi_{i}^{I}-\widetilde{\Phi}_{i}^{I}}{\alpha^{I}+1}+\widetilde{\Phi}_{i}^{\mathrm{ref}}$

restores the flux of image $I$ and superpixel $i$ to the photometric level of the reference image.

Since Eq. (1) is a statistical correlation with an intrinsic width, Eq. (2) implies a residual Gaussian noise which is constant over each image. The error bar on $\Phi_{i}^{\mathrm{C}, \mathrm{I}}$ is then redefined to be

$\sigma_{i}^{I}=\sqrt{\left(\sigma_{\gamma, i}^{I}\right)^{2}+\left(\sigma_{\mathrm{see}}^{I}\right)^{2}}$

where $\sigma_{\gamma, i}^{I}$ is the photon noise in the superpixel $i$ of image $I$ and $\sigma_{\text {see }}^{I}$ is the residual noise on image $I$. Figure 3 shows an example of seeing stabilisation for the lightcurve of a typical unresolved variable star. The larger the superpixel size, the larger the photon noise, since a larger bin introduces more background into the data. The superpixel size of $7 \times 7$ pixels $(2$ "' $31 \times 2$ '. 31$)$ is chosen because it gives the best compromise between seeing stabilisation and $S / N$ (Paulin-Henriksson 2002).

\subsection{Selection of microlensing candidates}

We fit all preselected lightcurves to a standard microlensing curve (Paczyński 1986) with seven parameters: the Einstein crossing time $t_{\mathrm{E}}$, the date of maximum magnification $t_{0}$, the impact parameter $u_{0}$, and two flux parameters for each filter, one for the source $F_{\mathrm{s}}$ and the other for the background $F_{\mathrm{b}}$. To allow for non-standard microlensing events, we initially set a loose threshold of $\chi^{2} /$ d.o.f. $<5$. To keep high $S / N$ candidates, we calculate the probability $P$ that a bump is due to random noise, and demand $-\ln P>100$ in $r^{\prime}$ and $-\ln P>20$ in one other filter, with at least two points (in either band) on both the rising and falling parts of the variation. These cuts leave 441 candidates, one third of which show secondary peaks. Our purpose here is to present events for which we have very high confidence. To eliminate periodic variables, we reject by eye every lightcurve with a secondary peak comparable (in terms of amplitude and shape) to the microlensing candidate. We then check if the remaining secondary peaks are due to variations
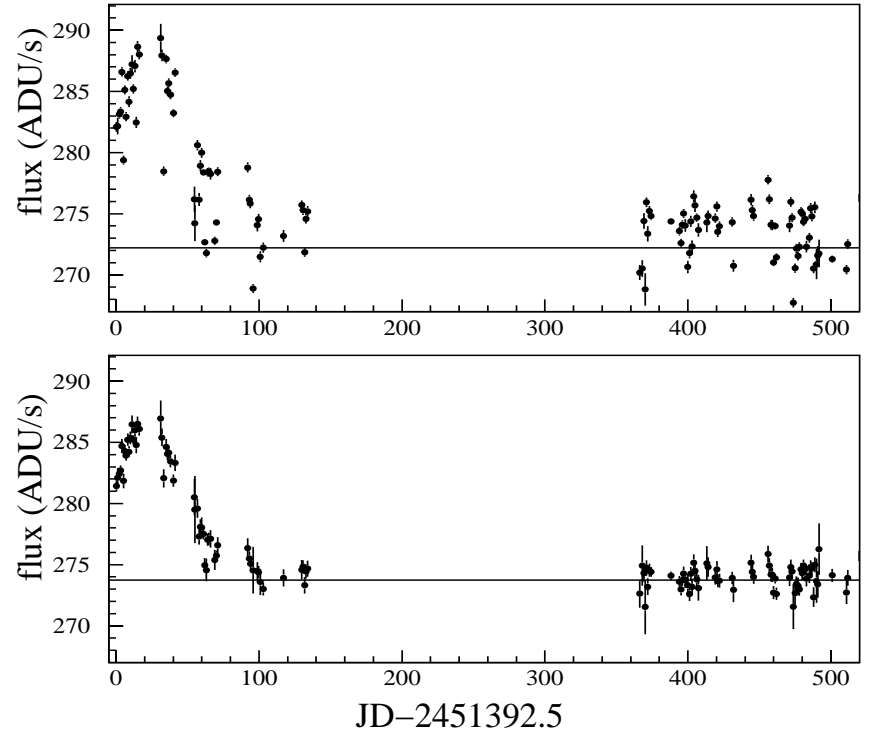

Fig. 3. Lightcurve in $r^{\prime}$ filter of a $7 \times 7$-superpixel centred on an unresolved variable star before (upper panel) and after (lower panel) seeing stabilisation. The horizontal line is the baseline.

in the neighbourhood with a simple image differencing test: for each peak, we add all images belonging to the peak and subtract as many images far from any peak and with similar seeing. On this difference image, variations separated by more than $\sim 1^{\prime \prime}(\sim 3$ pixels) are easily resolved, as shown for example in Sect. 3.2. If we cannot distinguish secondary bumps from the microlensing candidate, the lightcurve is rejected.

After this cull, there remain 362 candidates. To distinguish microlensing events with long full width at half maximum timescales $\left(t_{1 / 2}>25\right.$ days $)$ from intrinsically variable stars will require additional baseline data. These will need to come from a third season of observations and possibly from other telescopes, such as the MDM-McGraw-Hill telescope (Calchi Novati et al. 2002). Moreover, unless typical MACHO masses exceed $1 M_{\odot}$, we expect more than $80 \%$ of the microlensing events to have $t_{1 / 2}<25$ days. We therefore restrict ourselves to events shorter than 25 days. We expect this cut to eliminate most of the Mira variables. Noda et al. (2002) show that some Mira-like variables have large amplitudes and hence small $t_{1 / 2}$ and so may pass this cut. However, such variables have periods of the order of 100 days and so they will almost always have multiple bumps over our 2 years baseline. Then the probability for such a star to mimic a Paczyński curve is extremely small. This leaves eight candidates, four of which are discussed below. Of the other four events, one is suggestive of a binary lens and will be the subject of further analysis. Another shows some asymmetric correlations among the residuals of the Paczyński fit and is suspected to be due to a variable star rather than a microlensing effect. The two remaining events are not convincing microlensing candidates because they are too poorly sampled and/or have too noisy a baseline to allow the correlations to be studied. 
Table 1. Main characteristics of the four microlensing candidates. $d$ : distance from the centre of M $31 . t_{1 / 2}$ : full width at half maximum timescale. $t_{0}$ : date of maximum magnification. $u_{0}$ : impact parameter in units of the Einstein radius. $A_{\max }:$ maximum magnification $\left(\sim 1 / u_{0}\right.$ for such large magnifications). $t_{\mathrm{E}}$ : Einstein crossing time. For PA-99-N2, PA-00-S3 and PA-00-s4, $u_{0}, A_{\max }$ and $t_{\mathrm{E}}$ are computed without any prior on the source fluxes. The source star of PA-99-N1 has been identified on HST archival images (Aurière et al. 2001). $\Delta R$ : Cousins magnitude of the maximum source flux variation, which is also the magnitude at $t_{0}$ for such large magnifications. $V, R, I$ : Johnson/Cousins magnitudes. $S / N$ : total signal-to-noise ratio, as defined in Eq. (4); the number quoted in parenthesis indicates the number of points (in $r^{\prime}$ filter) contributing to $S / N$. $\chi^{2} /$ d.o.f.: $\chi^{2}$ per degree of freedom of the Paczyński fit; the number quoted in parenthesis indicates the number of degree of freedom of the fit.

\begin{tabular}{ccccc}
\hline \hline & PA-99-N1 & PA-99-N2 & PA-00-S3 & PA-00-S4 \\
\hline$\alpha(\mathrm{J} 2000)$ & $00 \mathrm{~h} 42 \mathrm{~min} 51.42 \mathrm{~s}$ & $00 \mathrm{~h} 44 \mathrm{~min} 20.81 \mathrm{~s}$ & $00 \mathrm{~h} 42 \mathrm{~min} 30.51 \mathrm{~s}$ & $00 \mathrm{~h} 42 \mathrm{~min} 29.97 \mathrm{~s}$ \\
$\delta(\mathrm{J} 2000)$ & $41^{\circ} 23^{\prime} 53.7^{\prime \prime}$ & $41^{\circ} 28^{\prime} 45.2^{\prime \prime}$ & $41^{\circ} 13^{\prime} 04.9^{\prime \prime}$ & $40^{\circ} 53^{\prime} 47.1^{\prime \prime}$ \\
$d$ & $7^{\prime} 52^{\prime \prime}$ & $22^{\prime} 03^{\prime \prime}$ & $4^{\prime} 00^{\prime \prime}$ & $22^{\prime} 31^{\prime \prime}$ \\
$t_{1 / 2}$ (days $)$ & $1.80 \pm 0.22$ & $21.75 \pm 0.20$ & $2.18 \pm 0.14$ & $2.09 \pm 0.11$ \\
$t_{0}(\mathrm{JD}-2451392.5)$ & $13.87 \pm 0.06$ & $71.56 \pm 0.10$ & $458.35 \pm 0.02$ & $488.90 \pm 0.07$ \\
$u_{0}$ & $0.057 \pm 0.004$ & $0.075 \pm 0.004$ & $0.053_{-0.016}^{+0.024}$ & $0.00472_{-0.00466}^{+0.00618}$ \\
$A_{\max }$ & $17.54_{-1.15}^{+1.33}$ & $13.33_{-0.67}^{+0.75}$ & $18.88_{-5.89}^{+8.15}$ & $211_{-120}^{+16456}$ \\
$t_{\mathrm{E}}$ (days) & $9.74 \pm 0.70$ & $91.91_{-3.83}^{+4.18}$ & $12.56_{-3.23}^{+4.53}$ & $128.58_{-72.27}^{+142.61}$ \\
$\Delta R$ & $20.80 \pm 0.13$ & $19.0 \pm 0.2$ & $18.8 \pm 0.2$ & $20.7 \pm 0.2$ \\
$V-R$ & $1.2 \pm 0.2$ & $1.0 \pm 0.1$ & & $0.0 \pm 0.1$ \\
$R-I$ & & & $0.6 \pm 0.1$ & $116.2(10 \mathrm{pts})$ \\
$S / N$ & $63.6(7 \mathrm{pts})$ & $1603.0(54 \mathrm{pts})$ & $115.7(5 \mathrm{pts})$ & $0.7(146 \mathrm{~d} . \mathrm{f})$. \\
$\chi^{2} /$ d.o.f. & $0.9(65$ d.f. $)$ & $3.1(233$ d.f. $)$ & $1.1(172 \mathrm{~d}$.f. $)$ & \\
\hline
\end{tabular}

\section{Four robust candidates}

The four candidates are PA-99-N1, PA-99-N2, PA-00-S3 and PA-00-S4. The letter N(S) indicates whether the event lies in the north(south) INT WFC field, the first number 99(00) gives the year in which the maximum occurs and the second number is assigned sequentially in the order of detection. Candidates PA-99-N1 and PA-00-S4 have already been discussed by Aurière et al. (2001) and Paulin-Henriksson et al. (2002).

The statistical relevance of the candidates is estimated via the total signal-to-noise ratio $S / N$ of the bumps in the $r^{\prime}$ filter:

$S / N=\sum_{i \in \text { bump }} \frac{\Phi_{i}-\Phi_{\mathrm{bl}}}{\sigma_{i}}$

where $\phi_{\mathrm{bl}}$ is the baseline flux given by the Paczyński fit. The sum is taken over all datapoints that lie more than $3 \sigma$ above the baseline. The four candidates have signal-to-noise ratios of between $\sim 60$ and $\sim 1600$, that is, high compared to typical events in the database. Table 1 gives the main characteristics of the four candidates, while Fig. 4 shows their lightcurves. In the following, they are presented in ascending order of distance to the centre of M 31, as illustrated in Fig. 5.

\subsection{PA-00-S3}

PA-00-S3 lies $4^{\prime} 00^{\prime \prime}$ from the centre of M 31, within the fields observed using the $1.3 \mathrm{~m}$ MDM-McGraw-Hill telescope (Calchi Novati et al. 2002). The continuation of the lightcurve in 1998 and 1999 with data from this telescope shows no secondary bump, confirming the microlensing hypothesis. At this position, bulge-bulge lensing is expected to dominate (e.g., Kerins et al. 2001). The high $S / N$ of this event, together with the fact that the wings of the lightcurve are well sampled, has enabled a remarkably good direct estimate of the Einstein crossing time, $t_{\mathrm{E}} \sim 13 \pm 4$ days. For comparison, we would expect timescales of $\sim 20\left(M / M_{\odot}\right)^{1 / 2}$ days for bulge-disk lensing, $\sim 30\left(M / M_{\odot}\right)^{1 / 2}$ days for bulge-bulge lensing or $\sim 60\left(M / M_{\odot}\right)^{1 / 2}$ days for disk-disk lensing. The proximity of PA-00-S3 to the bulge, together with its Einstein crossing time, strongly suggests that it is a low mass stellar lens event.

\section{2. $P A-99-N 1$}

PA-99-N1 lies 7'52" from the centre of M 31 and occurred in August 1999, at the beginning of the first season of observation. The data up until November 1999 have already been presented by Aurière et al. (2001). Since then, the baseline has been extended to January 2001, yielding better constraints on the parameters of the Paczyǹski fit (see Table 1). This baseline shows two secondary bumps in December 1999 and November-December 2000 but, using the simple image differencing procedure explained in Sect. 2.2, one can show that these secondary bumps are separated from the event by about 3 pixels $\left(\sim 1^{\prime \prime}\right)$, as shown in Fig. 6. Therefore, the existence of these secondary bumps is no reason to reject PA-99-N1, and data points belonging to secondary bumps are masked for further analysis.

Our detailed study of this event (Aurière et al. 2001) led us to conclude that the source star is almost certainly 

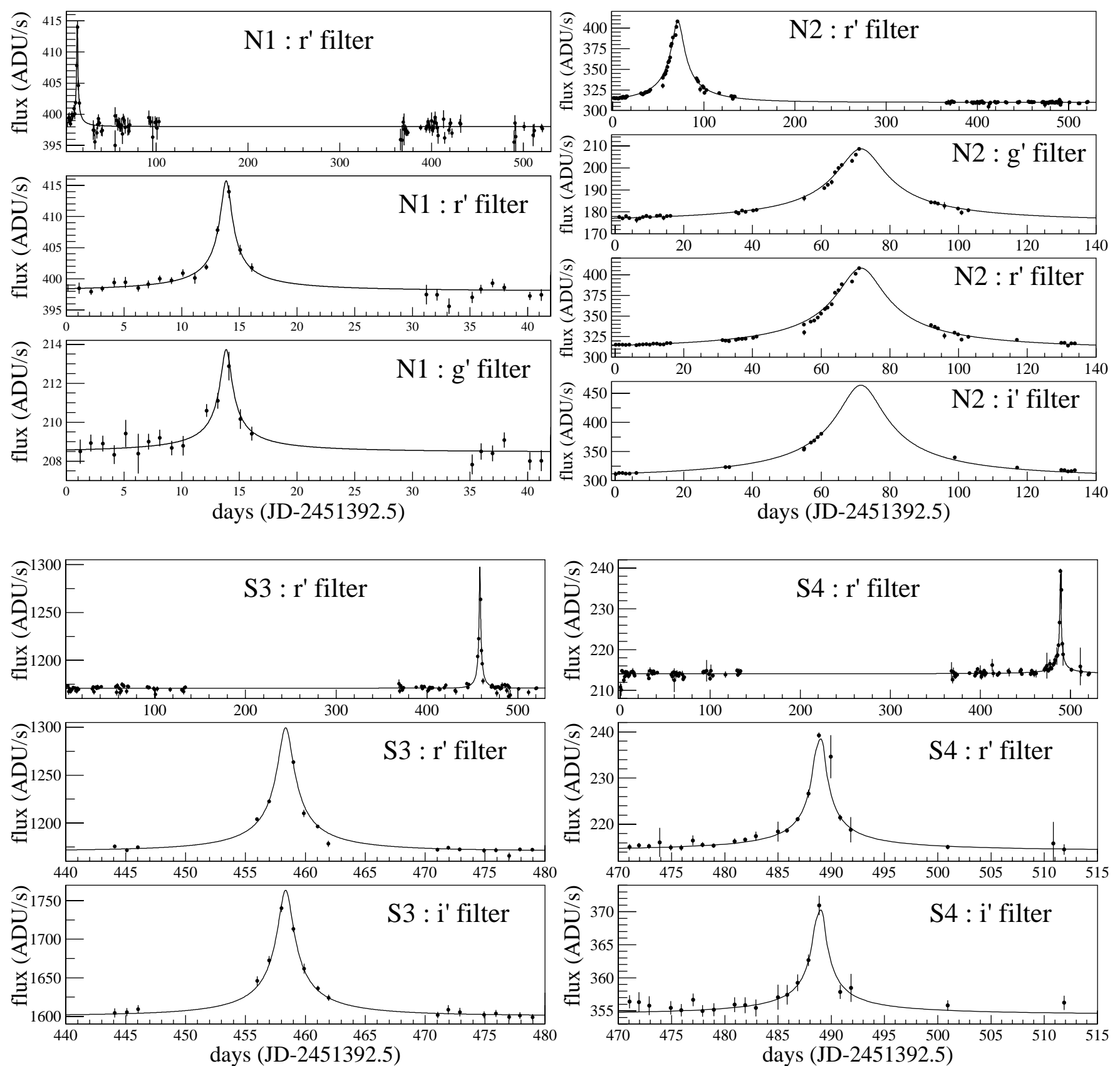

Fig. 4. Lightcurves of the four microlensing candidates (presented in Sects. 3.1 to 3.4). For each event, the top panel shows two seasons of analysed data in the $r^{\prime}$ filter. Lower panels are zooms that focus on candidates in all bands for which data have been taken. Solid lines are best-fit Paczyński (1986) curves. For PA-99-N1, secondary bumps due to a neighbouring variable star are masked (see Sect. 3.2). Note that deviations from the Paczyński curve for PA-99-N2 are achromatic (see Sect. 3.3).

identified on Hubble Space Telescope (HST) archival images and has Johnson/Cousins magnitudes: $I=22.41 \pm 0.10$, $V=24.51 \pm 0.12$. This allows one to break the degeneracy between the Einstein crossing time and the impact parameter, and so obtain direct measurements of the event duration and impact parameter: $t_{\mathrm{E}}=9.74 \pm 0.70$ days, $u_{0}=0.057 \pm 0.004$. These values are slightly different from those given in Auriere et al. (2001), as we have subsequently extended the baseline for this event. If the halo fraction is above $20 \%$, the lens is most probably a MACHO (with equal chance to be in the
M 31 or Milky Way halo). However, it is also plausible that the lens is a star, in which case the most probable mass is around $M \sim 0.2 M_{\odot}$.

\section{3. $P A-99-N 2$}

At $22^{\prime} 03^{\prime \prime}$ from the centre of M 31, PA-99-N2 lies well outside the projected area of the M 31 bulge and therefore appears to be an excellent candidate for a MACHO lens. Precise determination of all five parameters of the Paczyński fit is possible 


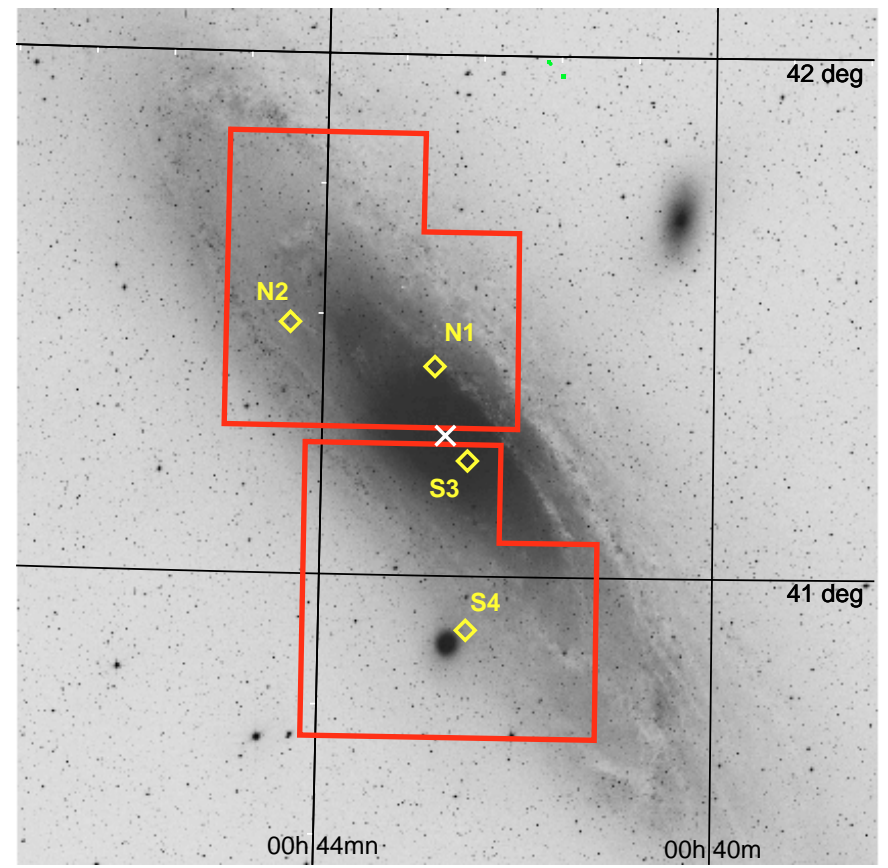

Fig. 5. Positions of the four microlensing candidates projected on M 31 (http://aladin.u-strasbg.fr, POSSII). The solid frames show the boundaries of observed fields and the white cross indicates the position of the centre of M 31. Note that S4 lies just next M 32 .

because of very good sampling and small error bars. We find a duration for this events of $t_{\mathrm{E}} \sim 92 \pm 4$ days and maximum magnification $A_{\max }=13.3 \pm 0.7$. MACHOs are expected to have a duration centred on $t_{\mathrm{E}} \sim 50\left(M / M_{\odot}\right)^{1 / 2}$ days. The alternative to MACHOs is self-lensing by an M 31 disk star (Gould 1994), with a typical duration $t_{\mathrm{E}} \sim 60\left(M / M_{\odot}\right)^{1 / 2}$ days.

The very high $S / N$ allows achromatic deviations to the standard Paczyński curve to be revealed, implying a $\chi^{2}$ per degree of freedom of 3.1. Some evidence of this is visible in Fig. 4. If these deviations are not simply due to systematic photometric errors, they are suggestive of a parallax effect (Alcock et al. 1997) and/or a close caustic approach (Albrow et al. 2002).

\subsection{PA-00-S4}

We have discussed previously PA-00-S4 (Paulin-Henriksson et al. 2002). It lies $22^{\prime} 31^{\prime \prime}$ from the centre of M 31, but unlike PA-99-N2, there is a concentration of stellar lenses along the line of sight. Indeed, it is only $2^{\prime} 54^{\prime \prime}$ from the centre of M 32. The blue color $(R-I=0.0 \pm 0.14)$ of this event argues strongly for a source that lies in the disk of M 31. Because M 32 is believed to be in the foreground (Byrd 1976; Ford et al. 1978), the proximity of the lens to the line of sight of M 32 suggests that it is most naturally interpreted as a star in M 32. However, this argument is not conclusive and a MACHO interpretation is still possible.

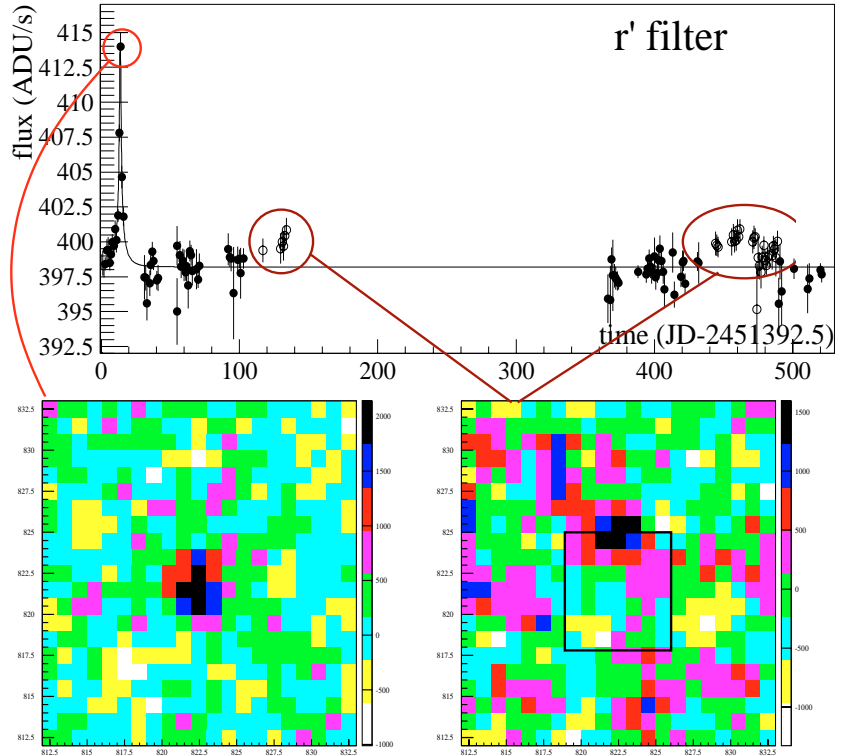

Fig. 6. Top panel: $r^{\prime}$-lightcurve of the PA-99-N1 microlensing candidate between August 1999 and January 2001. Encircled variations show three bumps, the first one being the microlensing candidate. As in Fig. 4, the solid line shows the best-fit Paczyński curve (data points for the secondary bumps being masked for this fit). Bottom panels: image differencing (at left around the maximum magnification of the microlensing event, at right on data points belonging to secondary bumps) showing that the microlensing event and the secondary bumps are separated by $\sim 3$ pixels $\left(\sim 1^{\prime \prime}\right)$. On the bottom right panel, a black square shows the superpixel centred on the microlensing candidate.

\section{Conclusion}

We have reported first results extracted from two years of data in our pixel lensing survey of the Andromeda galaxy. By imposing the stringent requirements $R(\Delta F)<21$ and $t_{1 / 2}<25$ days, we have selected four very convincing microlensing candidates with high signal-to-noise ratio and short timescales. For three of our four events we have been able to make reliable determinations of the Einstein crossing time, which provides additional clues as to the probable origin of these events. In the case of PA-00-S3, the event is most likely caused by a stellar lens in the M 31 bulge. In the three other cases, MACHOs and stellar lensing are plausible.

Acknowledgements. YLD was supported by a PPARC postdoctoral fellowship and SJS by a PPARC advanced fellowship. NWE acknowledges help from the Royal Society. Work by AG was supported in part by a grant from the Centre National de la Recherche Scientifique and in part by grant AST 02-01266 from the NSF.

\section{References}

Albrow, M. D., An, J., Beaulieu, J.-P., et al. 2002, ApJ, 572, 1031

Alcock, C., Allsman, R. A., Alves, D. R., et al. 1997, ApJ, 479, 119

Alcock, C., Allsman, R. A., Alves, D. R., et al. 2000, ApJ, 542, 281

Ansari, R., Aurière, M., Baillon, P., et al. 1997, A\&A, 324, 843

Ansari, R., Aurière, M., Baillon, P., et al. 1999, A\&A, 344, L49 
Aurière, M., Baillon, P., Bouquet, A., et al. 2001, ApJ, 553, L137

Baillon, P., Bouquet, A., Giraud-Héraud, Y., \& Kaplan, J. 1993, A\&A, 277,1

Byrd, G. G. 1976, ApJ, 208, 688

Calchi Novati, S., Iovane, G., Marino, A. A., et al. 2002, A\&A, 381, 848

Crotts, A. P. S. 1992, ApJ, 399, L43

Crotts, A. P. S., \& Tomaney, A. B. 1996, ApJ, 473, L87

Ford, H. C., Jacoby, G. H., \& Jenner, D. C. 1978, ApJ, 223, 94

Gondolo, P. 1999, ApJ, 510, L29

Gould, A. 1994, ApJ, 435, 573

Gould, A. 1996, ApJ, 470, 201
Haiman, Z., Magnier, E., Lewin, W. H. G., et al. 1994, A\&A, 286, 725

Kerins, E. J., Carr, B. J., Evans, N. W., et al. 2001, MNRAS, 323, 13

Lasserre, T., Afonso, C., Albert, J. N., et al. 2000, A\&A, 355, L39

Noda, S., Takeuti, M., Abe, F., et al. 2002, MNRAS, 330, 137

Paczyński, B. 1986, ApJ, 304, 1

Paulin-Henriksson, S. 2002, Ph.D. Thesis, Univ. Paris VI (in French) http://tel.ccsd.cnrs.fr/documents/archives $0 / 00 / 00 /$ 26/98/index_fr.html

Paulin-Henriksson, S., Baillon, P., Bouquet, A., et al. 2002, ApJ, 576, L121

Perryman, M., Lindegren, L., Kovalevsky, J., et al. 1997, A\&A, 323, L49 